\title{
WEB RESOURCE USAGE CONTROL IN RSCLP
}

\author{
Steve Barker
}

\begin{abstract}
We introduce a new usage control model for protecting Web resources. We also describe specification and implementation languages for policies that are defined in terms of our usage control model.
\end{abstract}

\section{INTRODUCTION}

The Web includes a number of different types of resources (e.g., software modules, databases, XML documents, etc.) that may need to be accessed, combined, and automatically processed by agents (i.e., software agents and human users). Nevertheless, not all of the content of all the heterogeneous resources that form part of the Web should necessarily be usable, observable, or modifiable by all agents. In P2P, B2C and B2B applications, fine-grained access to Web resources needs to be flexibly specified for different agents. For example, when a bank or an insurance company offers services to its customers, not all customers should be permitted to know about all of the services the company offers, and should not be able to access all of the documents that the company has. Access to resources on the Web may depend on the classification of a customer, e.g., whether a customer is a "preferred customer" or a "bad debtor". What is more, when one business requests information, about a service that another business provides, the information supplied to the requester, on perhaps things like discounting policies, refund policies, and lead times, will depend on a usage control policy in force. The usage control policy specifies what the requester agent is permitted to know about the requestees' resources.

In recent years, a number of researchers have developed some sophisticated usage control models (see, for example, [1], [9], [3], and [2]). Although each of these proposals has its attractions for protecting information in centralized systems and each could be enhanced to make it suitable for application in the case of usage control in an environment with heterogeneous distributed systems, we propose a new form of usage control model that is well suited for protecting Web-based resources. Our usage control model is expressed as an executable logic specification. 
The usage control model that we introduce is based on status levels (defined below). The status levels are assigned to agents that request access to system resources (henceforth these agents are referred to as requester agents), and to the resources themselves. A requester agent's status changes in a dynamic manner in response to the actions the requester agent performs. A mediating agent is used to evaluate usage requests from requester agents. The beliefs the mediating agent has about a requester agent's status are dynamically modified to take into account the requester agent's transaction patterns and behaviors (i.e., the mediating agent is a reactive agent). Moreover, the status assigned to a resource may change dynamically as a consequence of the occurrence of events. For example, the last tick of a clock at the end of a month may signal the downgrading of the status of a document from a classified status to an unclassified status. Because status levels are centrally important in the usage control model that we propose, we henceforth refer to the model as the Statusbased Usage Control (SBUC) model. We also describe an example SBUC policy in the sequel.

In previous work on the use of logic for access policy specification (see, for example, [9] and [2]), dynamic authorizations are not given emphasis. These previous works have been concerned with representations of access control models, discretionary access control (DAC) and role-based access control $(R B A C)$ models [6], for use in centralized systems. While DAC and RBAC are suitable for protecting resources in relatively static and centralized systems that are managed by human policy administrators, DAC and RBAC need to be generalized for the distributed case. SBUC generalizes DAC and RBAC, and can be used both for centralized and decentralized systems that may be static or highly dynamic in nature, and that may be managed by humans or autonomously.

In addition to proposing a new usage control model that is based on dynamic authorization management, we also challenge the standard assumption that the operational methods that are used to evaluate access requests are required to produce a simple yes/no (true/false, allowed access/not allowed access, ...) response. Instead, in our approach a response to a usage request may generate factual information that the requester is permitted to see (e.g., the requester's bank balance), or a conditional answer (e.g., a rule-based policy specification), or a yes/no answer (if a request is simply to know whether a fact is true in a world that is described by some remote data store).

$S B U C$ policies are specified by using a language that we refer to as RSCLP (defined below). RSCLP is short for Restricted SCLP. That is, we propose using a restricted form of the recently proposed Situated Courteous Logic Programming (SCLP) language [7], a programming language that permits procedural attachments [7] to be invoked from logic programs in order to support e-business applications. Our RSCLP language does not include the 
use of effectors or rule prioritization, both of which are key elements of SCLP. Rather, we restrict our attention to SCLP sensors, and we use these sensors in a controlled fashion that ensures that we do not compromise the formal semantics that we choose to adopt for $S B U C$ policy specifications (i.e., the Well Founded Semantics [13]). By ensuring that we have a sound formal basis for our $S B U C$ model and policies, we preserve one of the major attractions of using logic for specifying usage control policies, and we satisfy one of the essential requirements for all usage control models.

For policy information to be shared by multiple agents in the framework that we propose, we envisage using RuleML [4]. RuleML is an inter lingua that enables a variety of current commercially important (CCI) systems [7] to interoperate. Formulating $S B U C$ policies in $R S C L P$, produces a specification with respect to which usage requests on Web resources may be evaluated by using well known and efficient (i.e., PTIME) operational semantics. By employing RuleML, with $S B U C$ policies represented using $R S C L P$, we enable resources to be shared in a controlled fashion between agents that use different CCI systems to store and process information.

In work related to ours, XACML [10] has recently been proposed as a candidate for usage control specification for information resources on the Web. Nevertheless, some problematic issues arise with XACML. For instance, the low-level/procedural/hard-coded nature of XACML is inappropriate for the policy specifiers of the future (i.e., lawyers, accountants, etc. rather than computer scientists), the use of additional functions of arbitrary complexity may make the effects of XACML policies difficult for policy authors to understand, and XACML is based on a policy-level perspective rather than being based on a (higher-level) usage control model perspective (albeit profiles [10] may be used to relate XACML policy formulations to usage control models). Tools will be required in order for policy specifiers to formulate usage control policies using XACML, but that requires policy authors to use additional software systems. In our approach, $R S C L P$ is used as a high-level usage policy specification language that enables a number of emerging Web notions (e.g., ontologies [8]) to be employed in usage control.

Space restrictions prevent us from considering authentication issues in this paper. Rather, we assume that agents have been authenticated prior to submitting usage requests on Web resources. We do not consider trust issues, and we do not consider the issue of securing data by encryption. Our approach can, however, naturally accommodate notions like digital identification, and trustbased usage control. We also restrict our attention to retrievals of factual and policy information from remote data sources.

The rest of the paper is organized thus. In Section 2, some basic notions are briefly described. In Section 3, we define a restricted form of the $S B U C$ model, and we describe a specialization of the $S B U C$ model for represent- 
ing an application-specific $S B U C$ policy. In Section 4, we give an example of an $S B U C$ policy formulation, and we consider some practical issues relating to our approach. Finally, in Section 5, some conclusions are drawn and suggestions for further work are made.

\section{PRELIMINARIES}

The $S B U C$ model and the $S B U C$ policies that we describe in later sections are expressed in terms of our RSCLP language. Principally, our RSCLP language includes (i) normal clauses (with one type of negation), and (ii) sensor statements.

DEFINITION 1 A normal clause is a formula of the form:

$$
C \leftarrow A_{1}, A_{2}, \ldots, A_{m}, \text { not } B_{1}, \text { not } B_{2}, \ldots, \text { not } B_{n} \quad(m \geq 0, n \geq 0) .
$$

The head, $C$, of the clause above is a single atom. The body of the clause (i.e., $A_{1}, A_{2}, \ldots, A_{m}$, not $B_{1}$, not $B_{2}, \ldots, n o t B_{n}$ ) is a conjunction of literals. Each $A_{i}$ literal $(i \in\{1, . ., m\})$ is a positive literal; each not $B_{j}$ literal $(j \in$ $\{1, \ldots, n\})$ is a negative literal. In the case of a negative literal in RSCLP, the relevant type of negation is negation as failure [5].

DEFINITION 2 A sensor statement has the form:

$$
C:: s:: P
$$

where $C$ is an "ordinary predicate", and $P$ is an external procedure that may be expressed in a language that is outside of normal clause logic.

REMARK 1 A sensor statement is used to generate information that may be used to determine the truth value of an "ordinary predicate" [7].

The restricted form of sensor statements that we admit in RSCLP are used to evaluate (on positive integers):

- Comparison operators in the set $\Phi=\{<, \leq,=, \neq, \geq,>\}$; and

- Arithmetic operators in the set $\Psi=\{+,-, \div, \times, \bmod , a b s\}$.

In our $S B U C$ model and $S B U C$ policies, certain predicates in the alphabet $\boldsymbol{\Sigma}$ of the RSCLP language that we use for specification have a fixed intended interpretation (see below). The only necessary terms in $\Sigma$ are constants and variables. Variables in RSCLP clauses will be denoted by using symbols

\footnotetext{
${ }^{1} \div$ is integer division
} 
that appear in the upper case, and constants will be denoted by lower case symbols. Although we later make use of function symbols in our specification of $S B U C$ policies, an equivalent function-free representation is possible. Hence, function-free $R S C L P$ is sufficient for specifying the SBUC model and $S B U C$ policies. In the sequel, we will refer to an $S B U C$ policy formulation in $R S C L P$ as an $S B U C$ program. An $S B U C$ program will include the rules that define the $S B U C$ model together with a set of rules that defines an application-specific $S B U C$ policy.

An $S B U C$ program $\psi$, henceforth written $S B U C(\psi)$, is defined on a domain of discourse that includes:

- A countable set $\mathcal{U}$ of requester agent identifiers such that $\mathcal{U}=\left\{u_{i}\right.$ : $\left.i \in \mathbb{N}_{C}\right\}$;

- A countable set $\mathcal{R}$ of resource identifiers such that $\mathcal{R}=\left\{r_{i}: i \in \mathbb{N}_{C}\right\}$;

- A countable set $\mathcal{P}$ of access privilege identifiers such that $\mathcal{P}=\left\{p_{i}\right.$ : $\left.i \in \mathbb{N}_{\subset}\right\} ;^{2}$

- A countable set $\mathcal{A}$ of action identifiers that may be performed by agents in an application-specific domain such that $\mathcal{A}=\mathcal{S}_{\mathbf{a}} \cup \mathcal{J}$ where $\mathcal{S}_{a}$ is a set of character strings that identify actions, and $\mathcal{J}=\left\{a_{i}: i \in\right.$ $\Psi\}$ where $\Psi \subseteq \mathbb{N}_{\subset},|\Psi|=\left|\mathbb{N}_{\subset}\right|-\left|\mathcal{S}_{a}\right|$;

- A countable set $\mathcal{L}$ of status level identifiers such that $\mathcal{L}=\left\{l_{i}: i \in\right.$ $\left.\mathbb{N}_{\subset}\right\}$

- A countable set $\mathcal{E}$ of event identifiers such that $\mathcal{E}=\left\{e_{i}: i \in \mathbb{N}_{\subset}\right\}$;

- A countable set $\mathcal{T}$ of time points such that $\mathcal{T}=\left\{t_{i}: i \in \mathbb{N}_{\subset}\right\}$.

We view time as a linearly ordered, discrete set of time points that are isomorphic to the set of natural numbers $\mathbb{N}$, and ordered by an "earlier than or the same time as" relation on $\mathcal{T}, \leq \mathcal{T}$. In practice, $\mathcal{T}$ is a bounded (finite) subset of $\mathbb{N}, \mathbb{N}_{C}$. We use $\mathbb{N}_{C}$ to denote any finite subset of $\mathbb{N}$, and we assume that times have a DAY granularity. ${ }^{3}$ Although we will assume that all times are valid times, our approach may be used to accommodate a variety of temporal dimensions.

DEFINITION 3 If $p_{n}$ is an access privilege $\left(p_{n} \in \mathcal{P}\right)$ and $r_{k}$ is a resource $\left(r_{k}\right.$ $\in \mathcal{R})$ then a permission is a pair $\left(p_{n}, r_{k}\right)$ that denotes that the $p_{n}$ access privilege is permitted on $\boldsymbol{r}_{k}$.

\footnotetext{
${ }^{2}$ In practice, access privileges will be named by character strings like read and write.

${ }^{3}$ The choice of the granularity of time (e.g., DAY, HOUR, ...) to be used in practical policies will be an application-specific one. The approach that we describe is not dependent on a specific choice of time granularity.
} 
In our proposal, sensors are only used for the implementation of a theory of Arithmetic that is used to manipulate the numbers that are used in SBUC policy formulations (and policy documents generally). This restricted use of sensors does not compromise the Well-founded Semantics for SBUC programs. The clauses that are included in the SBUC model are locally stratified [11], and we suggest that all realistic $S B U C$ policies may be expressed by using locally stratified logic. It follows that all "realistic" $S B U C$ programs are locally stratified. The following Proposition is important in this respect.

PROPOSITION 1 Every locally stratified program has a unique, 2-valued well-founded model.

In the sequel, we will denote the well-founded model of an $S B U C$ program $\psi$ by $W F M(S B U C(\psi))$.

\section{THE SBUC MODEL AND SBUC POLICY FORMULATION}

In this section, we give a brief overview of a simple form of the $S B U C$ model. Later in this section, we discuss some general notions relating to $S B U C$ policy formulation. However, we start by considering the basic factual information that is used in $S B U C$ programs to determine a user's status level.

\subsection{Security Event Descriptions}

In an SBUC program, a history of requester agent actions is used to determine the status an agent has at the time of the agent's usage request. These actions are expressed via a set of application-specific security event descriptions.

DEFINITION 4 A security event description is a finite set of ground 2-place assertions that describe an event and which includes four necessary facts and $n$ optional facts $(n \geq 0)$.

DEFINITION 5 The four necessary facts in a security event description $\epsilon$ together with their intended meanings are as follows (where $\psi$ is an SBUC program):

- $W F M(S B U C(\psi)) \vDash h a p p e n s\left(e_{i}, t_{j}\right)$ iff the event identified by $e_{i} \in \mathcal{E}$ happens at time $t_{j} \in \mathcal{T}$.

- $W F M(S B U C(\psi)) \vDash \operatorname{act}\left(e_{i}, a_{l}\right)$ iff the event identified by $e_{i} \in \mathcal{E}$ relates to an action $a_{l} \in \mathcal{A}$.

- $W F M(S B U C(\psi)) \vDash$ agent $\left(e_{i}, u_{m}\right)$ iff the event identified by $e_{i} \in \mathcal{E}$ relates to the requester agent $u_{m} \in \mathcal{U}$. 
- $W F M(S B U C(\psi)) \vDash$ resource $\left(e_{i}, r_{i}\right)$ iff the event identified by $e_{i} \in$ $\mathcal{E}$ relates to the resource $r_{i} \in \mathcal{R}$.

EXAMPLE 1 Consider the security event description

$$
\begin{array}{r}
\epsilon=\left\{\text { happens }\left(e_{1}, 12 / 12 / 2003\right) ; \text { agent }\left(e_{1}, \text { bob }\right) ; \text { act }\left(e_{1}, \text { depositing }\right)\right. \\
\text { resource } \left.\left(e_{1}, a_{1}\right) ; \text { amount }\left(e_{1}, 1000\right)\right\} .
\end{array}
$$

The set of facts in $\epsilon$ describes an event $\boldsymbol{e}_{1}$ that happens on 12/12/2003, and that involves the agent Bob depositing an amount of 1000 Euros into a resource (a bank account) denoted by $a_{1}$. The amount $\left(e_{1}, 1000\right)$ fact is the only non-necessary fact in $\epsilon$.

\subsection{The $S B U C$ Model}

In the $S B U C$ model, a partial ordering of elements in $\mathcal{L}$ is used to specify that agents and permissions that are assigned to a status level $l j$ are implicitly assigned to any status level $l_{i}$ that is "higher" in the partial order. As the hierarchical ordering of status levels is used for specifying both agent-status level and permission-status level assignments, we use the term assignment hierarchy to refer to the partial ordering on $\mathcal{L}$. The assignment hierarchy is defined in RSCLP in the following way (in which ' ', is an anonymous variable):

$$
\begin{aligned}
& \text { includes }(L 1, L 1) \leftarrow \operatorname{di}(L 1,-) . \\
& \text { includes }(L 1, L 1) \leftarrow \operatorname{di}(-, L 1) . \\
& \text { includes }(L 1, L 2) \leftarrow \operatorname{di}(L 1, L 2) . \\
& \text { includes }(L 1, L 2) \leftarrow \operatorname{di}(L 1, L 3), \text { includes }(L 3, L 2) .
\end{aligned}
$$

The extension of the irreflexive-intransitive $d i$ relation (where $d i$ is short for directly includes) comprises all pairs of status levels $\left\langle l_{i}, l_{j}\right\rangle\left(l_{i} \neq l_{j}\right)$ such that $\operatorname{WFM}(S B U C(\psi)) \models$ includes $\left(l_{i}, l_{j}\right)$, and there is no status level $l_{k}$ $\left(l_{i} \neq l_{k}, l_{j} \neq l_{k}\right)$ such that $W F M(S B U C(\psi)) \vDash \operatorname{includes}\left(l_{i}, l_{k}\right)$, and $W F M(S B U C(\psi)) \models$ includes $\left(l_{k}, l_{j}\right)$.

In the $S B U C$ model, a predicate sla with the following fixed meaning is used to define a user's status level:

- $W F M(S B U C(\psi)) \vDash s l a\left(u_{i}, l_{j}\right)$ iff the requester agent $u_{i} \in \mathcal{U}$ is assigned the status level $l_{j} \in \mathcal{L}$;

DEFINITION 6 The sla relation in $\operatorname{SBUC}(\psi)$ is defined thus:

$$
\begin{array}{r}
\text { sla }(U, L 1) \leftarrow \text { current_time }(T), \text { agent }(E 1, U), \text { happens }(E 1, T 1), \\
T 1 \leq T, \text { started_sla }(E 1, U, L 1), \\
\text { not ended_sla }(U, L 1, T 1, T) .
\end{array}
$$


Informally, the definition of sla specifies that a requester agent $U$ is assigned to the status level $L 1$ at the time $T$ of $U$ 's access request (where $T$ is the "current time" extracted from the system clock) if an event $E 1$ happens at a time $T 1$ that is earlier than or the same time as $T$, the occurrence of $E 1$ causes $U$ to be assigned to $L 1$, and $U$ 's assignment to $L 1$ has not been terminated in the interval $[T 1, T]$.

DEFINITION 7 The auxiliary started_sla predicate in sla is defined thus:

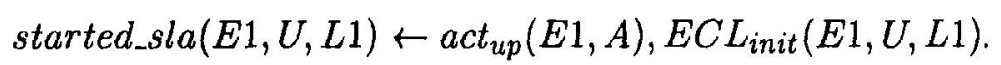

Informally, the definition of started_sla specifies that if an event $E 1$ involves an act $A$ that causes user $U$ 's status to be upgraded (expressed by the $a_{u p}$ atom), and the conditions expressed on $U$ 's assignment to $L 1$, as a consequence of $E 1$ happening, are satisfied then $U$ 's assignment to $L 1$ is started by the occurrence of $E 1$.

DEFINITION 8 The auxiliary ended_sla predicate in sla is defined thus:

$$
\begin{array}{r}
\text { ended_sla }(U, L 1, T 1, T) \leftarrow \operatorname{agent}(E 2, U), \text { happens }(E 2, T 2), \\
\operatorname{act}_{\text {down }}(E 2, A), E C L_{t e r m}(E 2, U, L 1), \\
T 1 \leq T 2, T 2 \leq T .
\end{array}
$$

Informally, the definition of ended_sla specifies that if, in the interval $[T 1, T]$, an event $E 2$ involves an act $A$ that causes user $U$ 's status to be downgraded (expressed by the $a_{c} t_{\text {down }}$ atom), and the conditions expressed on $U$ 's assignment to $L 1$, as a consequence of $E 2$ happening, are satisfied then $U$ 's assignment to $L 1$ is ended as a consequence of $E 2$ happening at the time $T 2$ in the interval $[T 1, T]$.

DEFINITION 9 The act ${ }_{u p}(E 1, A)$ clause is a clause of the form

$$
\operatorname{act}_{u p}(E 1, A) \leftarrow \operatorname{act}(E 1, A), \text { upgrading_act }(A) \text {. }
$$

DEFINITION 10 The act down $(E 2, A)$ clause is a clause of the form

$$
\operatorname{act}_{\text {down }}(E 2, A) \leftarrow \operatorname{act}(E 2, A) \text {, downgrading_act }(A) \text {. }
$$

There will be a fact, in $S B U C(\psi)$, of the form upgrading act $\left(a_{i}\right)$ for each upgrading act $a_{i} \in \mathcal{A}$, and a fact of the form downgrading_act $\left(a_{j}\right)$ for each downgrading act $a_{j} \in \mathcal{A}$. The sets of upgrading acts and downgrading acts are disjoint and application-specific.

\subsection{SBUC Policy Specification}

In the previous subsection we defined the $S B U C$ model. In this subsection, we consider $S B U C$ policy specification. 
To define the authorized forms of access that are expressed by an SBUC policy specification, we use an SBUC authorization clause. A variety of $S B U C$ authorization clauses may be represented to define different types of $S B U C$ policy. For a closed $S B U C$ policy [2], the following $S B U C$ authorization clause will suffice.

DEFINITION 11 The SBUC authorization clause (for closed usage policies) is as follows:

authorized $(U, P, R) \leftarrow \operatorname{sla}(U, L 1)$, includes $(L 1, L 2), \operatorname{pla}(P, R, L 2)$.

The $S B U C$ authorization clause in Definition 11 is used to specify that a user $u_{i} \in \mathcal{U}$ may exercise a privilege $p_{j} \in \mathcal{P}$ on a resource $r_{k} \in \mathcal{R}$ if $U$ is assigned to a status level $l_{m} \in \mathcal{L}$ and the permission $\left(p_{j}, r_{k}\right)$ is assigned to a status level $l_{n} \in \mathcal{L}$ such that includes $\left(l_{m}, l_{n}\right)$ holds.

The $E C L_{\text {init }}$ and $E C L_{\text {term }}$ atoms in the definitions of started_sla and ended_sla respectively specify the application-specific conditions on the actions of status level assignment and deassignment that arise as a consequence of the occurrence of events (i.e., ECL is short for event-condition-level; init is short for initiates; and term is short for terminates).

DEFINITION $12 E C L_{\text {init }}$ and $E C L_{\text {term }}$ are defined by clauses of the forms:

$E C L_{\text {init }}(E 1, U, L 1) \leftarrow A_{1}, A_{2}, \ldots, A_{m}$, not $B_{1}$, not $B_{2}, \ldots$, not $B_{n}$.

$E C L_{\text {term }}(E 1, U, L 1) \leftarrow A_{1}, A_{2}, \ldots, A_{m}$, not $B_{1}$, not $B_{2}, \ldots$, not $B_{n}$.

Due to space limitations, we only consider the simple form of pla given in the definition that follows.

DEFINITION 13 A permission-level assignment is expressed in $S B U C(\psi)$ by using clauses of the following form:

$$
\operatorname{pla}(P, R, L) \leftarrow A_{1}, A_{2}, \ldots, A_{m}, \text { not } B_{1}, \operatorname{not} B_{2}, \ldots, \text { not } B_{n} .
$$

\section{PRACTICAL SBUC POLICY SPECIFICATION IN RSCLP}

In practice, enterprises and individual users need to be able to exchange factual and policy information. Rather than sending complete fact and policy bases from one e-business $b_{1}$ to another e-business or customer $b_{2}, b_{1}$ may need to send a subset of the information it stores to $b_{2}$. The subset of policy information that is transferred from $b_{1}$ to $b_{2}$ will often depend on $b_{2}$ 's status as perceived by $b_{1}$. For instance, a "bad debtor" should not necessarily be sent the same information as a "preferred customer". In practice, it is important to be 
able to provide different agents with different views, depending on the agent's status, of the factual information and policy information that is stored on Web servers.

Although SBUC policy information about notions like agent locations (e.g., IP addresses), discrete trust levels, system status information, etc. are important for controlling agent access to Web resources and all of these elements may be represented by using numbers, in the sequel we will restrict our attention to the use of natural numbers for the practical representation of temporal information (viewed as "integer time values" [12]) in SBUC policies. Temporal constraints on resource usage are especially important in practice. Temporal constraints permit a policy author to specify that user access to a data item is to hold for a restricted interval of time, and automatically expires as soon as the maximum time point in the interval is reached, they provide a policy author with a fine level of control on assignments, they can limit the damage an unauthorized user may wreak if the user gains access to a system, they provide a revocation mechanism that is difficult to implement effectively in distributed systems that are managed by an autonomous agent, and they are important in workflow applications.

To represent times, we use integers of the form $y_{1} y_{2} y_{3} y_{4} m_{1} m_{2} d_{1} d_{2}$ : the $d_{1} d_{2}$ th day of the $m_{1} m_{2}$ th month of the $y_{1} y_{2} y_{3} y_{4}$ th year. Thus, $y_{1} y_{2} y_{3} y_{4}$ are the four year digits, e.g., 2004, $m_{1} m_{2}$ are the month digits, e.g., 11 (November), 03 (March), and $d_{1} d_{2}$ are the day of the month digits, e.g., 05. For example, 20040615 represents the 15 th of June 2004. All times are converted to this standard format in our approach (so differences between time formats can be compiled away and arithmetic operators like + and - may be used to resolve differences between time zones).

In an $S B U C$ policy, specified in RSCLP, sensor statements are used to associate a pure belief predicate [7] with an external procedure that is used to manipulate numbers/times. We envisage that these number/time manipulating procedures will be globally available (as ontologies) and will therefore be shareable by all applications that use $S B U C$ policies to protect resources. In addition to making use of sensor statements, URIs will be used to identify resources that may be accessed by agents from remotely located servers, and that may be transmitted from a business $b_{1}$ to a business $b_{2}$ depending on (i) $b_{2}$ 's status as perceived by $b_{1}$, and (ii) the sensitivity of the resource in the case where the status of resources is specified in a formulation of an $S B U C$ policy. We envisage the factual or policy information, which may be represented on Web servers by using different CCIs, being converted into RuleML form for exchange between agents. 
EXAMPLE 2 Consider the following policy requirements, ${ }^{4}$ a user of an e-bank has a status $l_{1}$ if the user currently has a balance of at least 1500 Euros, has remained in creditfor the past 3 months, and has joined the bank's loyalty scheme on or subsequent to its launch on 30th April, 2003. Suppose too that all customers are assigned to the status level $\boldsymbol{h}_{2}$ (as a minimum). For the SBUC program in RSCLP we have:

$$
\begin{aligned}
& E C L_{\text {init }}\left(E, U, l_{1}\right) \leftarrow \text { resource }(E, R), \\
& \text { balance }(E, R, X) \text {, } \\
& X \geq 1500, \text { loyal }(U) \text {, } \\
& \text { current_time }(T) \text {, } \\
& \text { earlier_month }(T, 3, T 1) \text {, } \\
& \text { not broken }\left(E, U, R, l_{0}, T 1, T\right) \text {. } \\
& \operatorname{broken}\left(E, U, R, l_{1}, T 1, T\right) \leftarrow \text { happens }(E 1, T 2) \text {, } \\
& \text { within }_{C C}(T 2, T 1, T) \text {, } \\
& \text { act }(E 1 \text {, withdrawal }) \text {, } \\
& \text { resource }(E 1, R) \text {, } \\
& \text { balance }(E 1, R, X), X<0 \text {. } \\
& \text { loyal }(U) \leftarrow \text { happens }(E 2, T 3) \text {, } \\
& \text { act }(E 3, \text { joining }) \text {, } \\
& \text { resource }(E 3, \text { loyalty_scheme), } \\
& \operatorname{actor}(E 3, U) \text {, } \\
& \text { earlier_than_eq }(T 3,20030430) \text {. }
\end{aligned}
$$

For this application, we use the following sensors (where each external procedure is a Java method):

$$
\begin{aligned}
& \text { earlier_month }(X, Y, Z) \\
& :: s \text { :: utils.date.subtract }(X, Y, Z) . \\
& \text { within }{ }_{C C}(X, Y, Z) \\
& :: s \text { : utils.date.within }(X, Y, Z) . \\
& \text { earlier_than_eq }(X, Y) \\
& :: s \quad:: \text { utils.date.earlier_than_eq }(X, Y) . \\
& \quad l t(X, Y) \\
& \quad:: s:: \text { utils.arith.lt }(X, Y) .
\end{aligned}
$$




$$
\begin{aligned}
& \text { gteq }(X, Y) \\
& :: s:: \text { utils.arith.gteq }(X, Y) \text {. }
\end{aligned}
$$

For representing the scenario in Example 2, the earlier_month $(X, Y, Z)$ sensor is used to return a time $Z$ that is earlier than the time $X$ by $Y$ months. The within $_{C C}(X, Y, Z)$ sensor is used to return a Boolean value to denote whether $X$ is a time in the closed interval $[Y, Z]$. The earlier_than_eq $(X, Y)$ sensor is used to return a Boolean value to denote whether $X$ is an earlier time or the same time as $Y$. The $l t(X, Y)$ sensor is used to return a Boolean value to denote whether the number $X$ is less than the number $Y$. The $g t e q(X, Y)$ sensor is used to return a Boolean value to denote whether the number $X$ is greater than or equal to the number $Y$. The current_time $(T)$ predicate is used to extract the current time from the system clock at the site at which the usage request is evaluated.

EXAMPLE 3 Continuing with our running example, suppose that we have the following RSCLP specifications describing the e-bank's release of policy information on ticket pricing where different pricing policies are described in two resources $r_{1}$ and $r_{2}$ :

$$
\begin{array}{r}
\text { pla }\left(\text { retrieve,ticket_policy }\left(r_{1}\right), l_{1}\right) \leftarrow \text { current_time }(T), \\
\text { later_than_eq }(T, 20040101), \\
\text { earlier_than_eq }(T, 20040331) . \\
\text { pla(retrieve,ticket_policy } \left.\left(r_{2}\right), l_{2}\right) \leftarrow \text { current_time }(T), \\
\text { later_than }(T, 20040131) .
\end{array}
$$

For these specifications, the following sensors are needed in addition to those defined in Example 2:

$$
\begin{aligned}
& \text { later_than_eq }(X, Y) \\
& :: s \text { : utils.date.later_than_eq }(X, Y) . \\
& \text { later_than }(X, Y) \\
& :: s \text { :: utils.date.later_than }(X, Y) .
\end{aligned}
$$

In Example 3, the later_than_eq(X,Y) sensor is used to return a Boolean value to denote whether $X$ is a later time than the time $Y$ or the same time as $Y$. The later_than $(X, Y)$ sensor is used to return a Boolean value to denote whether $X$ is a later time than the time $Y$. 
From the application-specific SBUC policy, expressed using RSCLP in Example 2 and Example 3, and the formal specification of the $S B U C$ policy defined in Section 3, it follows that, by using any sound evaluation procedure, the policy information that is expressed via $r_{1}$ is available to requesters that are authorized to see these offers in the period from 1st January, 2004 until 31st March, 2004. Moreover, if includes $\left(l_{1}, l_{2}\right)$ holds, from the assignment hierarchy that is included in the definition of the SBUC policy, it follows that any agent that is assigned to $l_{1}$ is, as of 1st February, 2004, permitted to also see the policy information expressed via the resource $\eta_{2}$. Agents assigned to the $l_{2}$ status can access the policy information expressed in $\eta_{2}$ as of 1 st February, 2004. After 31st March, 2004, agents with $l_{1}$ status can only see the same policy information as agents with $l_{2}$ status. This is a consequence of the dynamic revocation of access to the $r_{1}$ resource to customers with $l_{1}$ status.

Some additional points should be noted:

- Although Example 3 above only considers access to policy information, access to other forms of information and other resources can be expressed in exactly the same way, and the same operational methods may be used to evaluate usage requests;

- SBUC policy information is separated from the resources that are stored on Web servers that may need to be accessed to answer usage requests. Hence, $S B U C$ policies and Web data may be updated independently of each other;

- Using SBUC, RSCLP and RuleML enables policies to be easily integrated even when policies have different features e.g., negative authorizations, conflict resolution strategies, authorizations clauses, etc. Moreover, using SBUC, RSCLP and RuleML as a common framework for usage policy representation, with an unambiguous, shared semantics, facilitates interoperability.

\section{CONCLUSIONS AND FURTHER WORK}

We have described an approach for usage control for Web resources in which $S B U C$ policies are formulated as RSCLP programs that are expressed in terms of a general usage control model, the $S B U C$ model. In the framework that we have described, access to Web resources is controlled according to agent and resource statuses. These statuses change dynamically as a consequence of the occurrence of events.

In planned future work, we intend to investigate the use of an extended form of $R S C L P$ for formulating $S B U C$ policies for Web resources where classical negation is appropriate. We also intend to investigate the use of RSCLP with 
constraint logic programming as a CCI system, and the use of SCLP itself for $S B U C$ policy formulation.

\section{References}

[1] S. Barker. Protecting deductive databases from unauthorized retrievals. In DBSec 2000, pages 301-311. Kluwer, 2000.

[2] S. Barker and P. Stuckey. Flexible access control policy specification with constraint logic programming. ACM Trans. on Information and System Security, 6(5):501-546, 2003.

[3] E. Bertino, B. Catania, E. Ferrari, and P. Perlasca. A system to specify and manage multipolicy access control models. In Proc. POLICY 2002, pages 116-127. IEEE Computer Society, 2002.

[4] H. Boley, S. Tabet, and G. Wagner. Design rationale of ruleml: A markup language for semantic web rules. In SWWS 2001, pages 381-401, 2001.

[5] K. Clark. Negation as failure. In H. Gallaire and J. Minker, editors, Logic and Databases, pages 293-322. Plenum, 1978.

[6] C. Date. An Introduction to Database Systems. Addison-Wesley, 2003.

[7] B. Grosof. Representing e-commerce rules via situated courteous logic programs in ruleml. Electronic Commerce Research and Applications, pages 2-20, 2004.

[8] J. Heflin and J. Hendler. Dynamic ontologies on the web. In Proc. 17th National Conference on Artificial Intelligence, pages 443-449, 2000.

[9] S. Jajodia, P. Samarati, M. Sapino, and V.S. Subrahmaninan. Flexible support for multiple access control policies. ACM TODS, 26(2):214-260, 2001.

[10] OASIS. extensible access control markup language (xacml), 2003. http://www.oasisopen.org/xacml/docs/.

[11] T. Przymusinski. On the declarative semantics of deductive databases and logic programming. In J. Minker, editor, Foundations of Deductive Databases and Logic Programming, pages 193-216. Morgan-Kaufmann, 1988.

[12] R. Snodgrass. The temporal query language tquel. ACM TODS, 12(2):247-298, 1987.

[13] A. van Gelder, K. Ross, and J. Schlipf. The well-founded semantics for general logic programs. JACM, 38:620-650, 1991. 